\title{
Resistance to anti-hormonal therapy in prostate cancer
}

\author{
Zoran Culig
}

Received: 2 April 2019 / Accepted: 29 May 2019 / Published online: 12 June 2019

(C) The Author(s) 2019

\begin{abstract}
Summary Advanced stage prostate cancer is frequently treated with androgen receptor antagonists. Improvement in patients' survival has been achieved with the anti-androgen enzalutamide. However, there may be an increasing number of point mutations of the androgen receptor during therapy. In addition, ligand-independent activation of truncated androgen receptors may occur during anti-hormonal therapy. In prostate cancer, there is also an increased expression of coactivators and decreased expression of corepressors, thus, contributing to the disease progression. Stromal factors such as interleukins also contribute to therapy resistance. Although preclinical studies with anti-interleukin- 6 antibodies opened new possibilities for treatment of prostate cancer, clinical trials have not demonstrated a survival benefit. Increased expression of glucocorticoid receptor has also been associated with advanced prostate cancer. Thus, one can consider the administration of glucocorticoid receptor antagonists in addition to anti-androgens. Stem cells have been described either after androgen treatment or androgen ablation or as a consequence of long-term use of drugs. Taken together, multiple experimental studies provided evidence on the mechanisms that limit usefulness of anti-androgens in prostate cancer.
\end{abstract}

Keywords Androgen receptor - Coregulatory proteins - Cytokines · Anti-androgens · Epithelial to mesenchymal transition

Professor Z. Culig, M.D. ( $\bowtie)$

Department of Urology, Medical University of Innsbruck, Innsbruck, Austria

zoran.culig@i-med.ac.at

\section{Take-home messages}

- Increase in androgen receptor (AR) expression may occur during long-term androgen ablation therapy or blockade of AR.

- Specific AR mutations were described after treatments with hydroxyflutamide, bicalutamide, and enzalutamide.

- Expression of AR coactivators may increase during androgen ablation therapy, thus, enhancing agonistic activities of anti-androgens.

- Interleukins and chemokines may play an important role in prostate cancer progression through suppression of apoptosis.

- Stem cells expression could also increase during androgen ablation therapy or chemotherapy.

- Innovative multidisciplinary clinical studies and reasonable use of bioinformatics are necessary to improve clinical treatment of advanced prostate cancer.

\section{Introduction}

Anti-hormonal therapy for prostate cancer is based on reduction of concentration of circulating androgens and/or blockade of androgen receptor (AR). The $\mathrm{AR}$ is present in prostate tissue as wild-type or truncated protein. The wild-type protein contains the $\mathrm{N}$-terminal region, which may have a different number of CAG repeats, the DNA-binding domain, and the ligand-binding domain. Research on truncated $\mathrm{AR}$ proteins has been the focus of studies on prostate cancer [1]. A lower number of CAG repeats is associated with a higher transcriptional activity of the receptor. Physiologically, the wild-type AR is activated by ligands (testosterone or dihydrotestosterone) and translocates to the nucleus thus regulating cellular proliferation, apoptosis, and differentiation. Induction of the prostate cancer biomarker prostate-specific 
antigen (PSA) by AR ligands is frequently considered an indicator of functional activity of the androgen signaling pathway. Either domain of the AR may interact with coactivators or corepressors, proteins which, following ligand binding, enhance or diminish AR activity. Alterations of coactivator and corepressor expression are frequently observed in human prostate cancer and will be discussed in more detail in this review.

Prostate cancer is increasingly treated not only by urologists but also by experts in medical oncology and radiotherapy. It is therefore important to summarize the most important mechanisms relevant to therapy failure. Alterations in AR activation are commonly investigated in such multidisciplinary studies which could provide some guidance for basic and translational research in the future.

\section{Anti-hormonal therapy in prostate cancer}

Numerous pharmacological approaches have been postulated and tested in prostate cancer. In general, these approaches may either target the AR in addition to any other molecule which is overexpressed in the disease. Earlier studies on AR blockade in prostate cancer were based on clinical therapy with hydroxyflutamide or bicalutamide. Those drugs antagonize ligand binding and cause a retardation of cancer cell proliferation. However, there is an obvious compensatory mechanism which is manifested by an increase in AR expression and activity during prolonged androgen ablation [2]. This type of AR regulation may occur in any case of androgen deprivation. More specific alterations are AR point mutations which were described mostly during development of endocrine therapy resistance. Thus, replacement of the amino acid valine at position 715 with methionine leads to increased activation by hydroxyflutamide and adrenal androgens [3]. Another anti-androgen bicalutamide may also induce specific mutations in patients tissue, thus, showing agonistic activity [4]. Clinically, these and similar findings may explain why some patients react to withdrawal of an anti-androgenic drug from therapy by decrease of PSA or clinical improvement. On the basis of all these findings, it was proposed that inhibition of AR expression may provide a benefit in multiple models of therapy-resistant prostate cancer [5]. However, clinical improvement in terms of patients' survival was achieved with the anti-androgen enzalutamide [6]. In functional experiments, it was demonstrated that the mutation F876L is causally responsible for acquisition of agonistic properties of enzalutamide [7]. In terms of clinical diagnostics, this mutation could be used to predict a therapy response to enzalutamide, provided that a sufficient number of clinical samples are available.

Taken together, it seems that AR mutations may occur with any anti-androgen treatment and that prostate cancer cells have developed adaptive mech- anisms in order to overcome the effects of androgen withdrawal or anti-androgen treatment.

\section{Clinically relevant AR coactivators}

There are a large number of coactivators and corepressors which interact with the AR. A potential difficulty in studies with coactivators is that a loss of one of them may cause a compensatory action of other regulatory proteins. AR coactivators are not specific and they could also regulate activity of other steroid receptors. In this context, it is worthwhile to mention transcriptional integrators p300 and CREBbinding protein (CBP) which are particularly important in prostate carcinogenesis. These proteins are up-regulated by androgen ablation and contribute to activation of the AR by ligands and non-steroidal compounds [8, 9]. These two proteins could also influence prostate cancer progression by up-regulating proliferation in cells which do not express the AR [10]. Another coactivator of major importance in prostate cancer is SRC-1 which is implicated in regulation of proliferation of androgen-sensitive cells and their derivatives representing castration therapyresistant prostate cancer [11]. Mechanistically, it was found that SRC-1 inhibits the expression of the tumor suppressor protein kinase D1 in prostate cancer cells, thus, contributing to prostate tumorigenesis [12]. AR coactivators are appropriate targets for novel experimental therapies, such as small molecules C646 or bromodomain inhibitors in various models of prostate cancer $[11,13]$. It is expected that therapeutic targeting will be improved in the future because of an increased number of patient-derived xenografts [14]. It is therefore possible that improved methodologies in laboratory diagnostics will lead to establishment of analytical methods for investigation of a larger number of coactivators that are overexpressed in prostate cancer [15]. Although most studies have focused on coactivators, it should be mentioned that corepressors such as REST may be inactivated in the disease [16]. It was shown that expression of REST is negatively correlated with disease progression. Further mechanistic studies in the area of coregulator inhibitors in prostate cancer are therefore of importance.

\section{Influence of microenvironmental cytokines on anti-hormonal therapy resistance}

It is well known that prostate stroma may significantly influence carcinogenesis, as a rich source of cytokines and chemokines. Some of these proteins have been studied by many research laboratories. There is a particular interest in interleukin-6 (IL6), a molecule highly expressed in advanced cancers. Interleukin-6 is implicated in regulation of AR activity [17]. Interleukin-6 regulates cellular events in prostate cancer through activation of the signaling pathway of Janus kinase/signal transducers and 
activators of transcription (STAT) factors which are increasingly phosphorylated in the disease. For this reason, molecules which target the STAT3 and AR pathways such as galiellalactone may be considered for future preclinical and clinical studies. It was shown that galiellalactone has a strong inhibitory effect on STAT3 in DU-145 xenografts [18]. In this context, the approach with anti-IL-6 antibodies has been tested in prostate cancer in vitro and in vivo [19, 20]. Interestingly, preclinical results have supported use of the antibody for future studies at the time when they were performed. On the other hand, clinical studies have been carried out with patients who previously received other therapies for prostate cancer [21, 22]. Those results suggest that clinical trials may consider in the future some important findings from experimental studies which include timing of therapy and drug combinations. One cannot exclude the fact that earlier administration of anti-IL-6 drugs in prostate cancer may lead to a more favorable clinical response.

In addition to IL-6, other cytokines such as IL8 have been implicated in therapy resistance and multiple mechanisms responsible for its effect have been described in the literature [23]. Progression towards enzalutamide therapy resistance could be facilitated in the presence of chemokine receptors such as CXCR7, as evidenced in several models [24]. Action of ILs is under control of endogenous suppressors of cytokine signaling and protein inhibitors of activated STAT. Although they have a role in the control of the JAK/STAT pathway they could at the same time cause cell cycle progression or inhibit apoptosis [25, 26].

Not only the AR but also other nuclear receptors are involved in prostate cancer progression. For example, in castration therapy-resistant prostate cancer there is an increased expression of glucocorticoid receptor [27]. Those results imply that therapeutic inhibition of the glucocorticoid receptors may be necessary to overcome inhibition of anti-hormonal therapy.

\section{Role of stem cells in anti-hormonal therapy}

For a long period of time, it has been postulated that increased stemness of prostate cancer cells which appears during therapy. Cellular stemness is associated with increased expression of molecules being responsible for epithelial-to-mesenchymal transition, such as N-cadherin or vimentin. Different results whether androgen ablation stimulates or inhibits epithelial-tomesenchymal transition in prostate cancer were published in the literature and the reasons for that have not been clarified so far [28, 29]. Increased stemness was observed in prostate cancer cells after long-term treatment with docetaxel, thus, pointing to appropriate selection of drugs and choosing additional targets [30].

\section{Conclusions and future directions}

This overview of mechanisms relevant to therapy failure in prostate cancer clearly shows that the mechanisms may be AR-dependent and -independent. Since multiple mutations of the AR have been described in patients' tissues, this fact opens the possibility for improved molecular diagnostics. Because of the nature of prostate cancer and clinical course, it may be important to identify these mutations in order to decide on the administration of an appropriate anti-androgen. It is also very important to assess the levels of oncogenes which are up-regulated during androgen ablation, in particular because of they could enhance AR activity. Anti-hormonal therapy in prostate cancer may be in addition associated with increased expression of truncated AR which do not need a ligand to become activated. Finally, there is increased evidence that stem cells which appear during therapy are difficult to target.

It may be however possible to improve the treatment depending on profiling of signaling pathways, identification of novel targets and design of novel pharmaceutical inhibitors, such as small molecule drugs. It should be therefore appreciated that there has been a major effort to improve prostate cancer modeling. Whereas in the past, only a small number of cell lines was available, there are new experimental procedures allowing us to study tumor biology with organoids, explants and patient-derived xenografts. Ongoing and future clinical trials should therefore exactly clarify advantages and disadvantages of different measurements of expression of truncated ARs in prostate cancer because their prognostic significance has not been completely established [31]. In this context, clinical trials in prostate cancer include the N-terminal domain inhibitors and may be particularly important to inhibit truncated constitutively active receptors [32]. Targeting endocrine therapy resistance may be also improved on the basis of the use of programmed cell death (PD)-1 inhibitors during progression on enzalutamide [33].

Taken together, it is necessary to discuss translational studies and clinical trials on the basis of findings summarized in the present review.

Funding Open access funding provided by University of Innsbruck and Medical University of Innsbruck.

Conflict of interest Z. Culig declares that he/she has no competing interests.

Open Access This article is distributed under the terms of the Creative Commons Attribution 4.0 International License (http://creativecommons.org/licenses/by/4.0/), which permits unrestricted use, distribution, and reproduction in any medium, provided you give appropriate credit to the original author(s) and the source, provide a link to the Creative Commons license, and indicate if changes were made. 


\section{References}

1. Ho Y, Dehm SM. Androgen receptor rearrangement and splicing variants in resistance to endocrine therapies in prostate cancer. Endocrinology. 2017;158:1533-42.

2. Kokontis J, Takakura K, Hay N, Liao S. Increased androgen receptor activity and altered c-myc expression in prostate cancer cells after long-term androgen deprivation. Cancer Res. 1994;54:1566-73.

3. Culig Z, Hobisch A, Cronauer MV, et al. Mutant androgen receptor detected in an advanced-stage prostatic carcinoma is activated by adrenal androgens and progesterone. Mol Endocrinol. 1993; 7:1541-50.

4. Hara T, Miyazaki J, Araki H, et al. Novel mutations of androgen receptor: A possible mechanism of bicalutamide withdrawal syndrome. Cancer Res. 2003;63:149-53.

5. Eder IE, Culig Z, Ramoner R, et al. Inhibition of LNCaP prostate cancer cells by means of androgen receptor antisense oligonucleotides. Cancer Gene Ther. 2000;7:997-1007.

6. Scott LJ. Enzalutamide: A review in castration-resistant prostate cancer. Drugs. 2018;78:1913-24.

7. KorpalM, KornJM, GaoX, etal. AnF876Lmutationinandrogen receptor confers genetic and phenotypic resistance to MDV3100 (enzalutamide). Cancer Discov. 2013;3:1030-43.

8. Debes JD, Schmidt LJ, Huang H, Tindall DJ. P300 mediates androgen-independent transactivation of the androgen receptor by interleukin 6 . Cancer Res. 2002;62:5632-6.

9. Comuzzi B, Nemes C, Schmidt S, et al. The androgen receptorco-activator CBPisup-regulated following androgen withdrawal and is highly expressed in advanced prostate cancer. J Pathol. 2004;204:159-66.

10. Santer FR, Höschele PP, Oh SJ, et al. Inhibition of the acetyltransferases p300 and CBP reveals a targetable function for p300 in the survival and invasion pathways of prostate cancer cell lines. MolCancer Ther. 2011;10:1644-55.

11. Agoulnik IU, Vald A, Bingman WE 3rd, et al. Role of SRC-1 in the promotion of prostate cancer cell growth and tumor progression. Cancer Res. 2005;65:7959-67.

12. Luef B, Handle F, Kharaishvili G, et al. The AR/NCOA1 axis regulates prostate cancer migration by involvement of PRKD1. Endocr Relat Cancer. 2016;23:495-508.

13. Jin L, Garcia J, Chan E, et al. Therapeutic targeting of the $\mathrm{CBP} / \mathrm{p} 300$ bromodomain blocks the growth of castrationresistant prostate cancer. Cancer Res. 2017;77:5564-75.

14. NavoneNM, vanWeerdenWM, Vessella RL, etal. Movember GAP1 PDX project: An international collection of serially transplantable prostate cancer patient-derived xenograft (PDX) models. Prostate. 2018;78:1262-82.

15. Liu S, Kumari S, Hu Q, et al. A comprehensive analysis of coregulator recruitment, androgen receptor function and gene expression in prostate cancer. Elife. 2017; https: / / doi. org/10.7554/eLife.28482.

16. Swensson C, Ceder J, Iglesias-Gato D, et al. REST mediates androgen receptor actions on gene repression and predicts early recurrence of prostate cancer. Nucleic Acids Res. 2014;42:999-1015.

17. Handle F, Puhr M, Schaefer G, et al. The STAT3 inhibitor galiellalactone reduces IL-6-mediated AR activity in benign and malignant prostate models. Mol Cancer Ther. 2018;17:2722-31.

18. Canesin G, Evans-Axelsson S, Hellsten R, et al. The STAT3 inhibitor galiellalactone effectively reduces tumor growth and metastatic spread in an orthotopic xenograft mouse model of prostate cancer. Eur Urol. 2016;69:400-4.

19. Steiner H, Cavarretta IT, Moser PL, et al. Regulation of prostate cancer cells selected in the presence of interleukin-
6 by the anti-interleukin- 6 antibody CNTO 328 . Prostate. 2006;66:1744-52.

20. Wallner L, Dai J, Escara-Wilke J, et al. Inhibition of interleukin-6 with CNTo 328, an anti-interleukin-6 monoclonal antibody, inhibits conversion of androgen-dependent prostate cancer to an androgen-independent phenotype in orchiectomized mice. Cancer Res. 2006;66:3087-95.

21. Dorff TB, Goldman B, Pinski JK, et al. Clinical and correlative results of SWOG S0354. A phase II trial of CNTO 328 (Siltuximab), a monoclonal antibody against interleukin6 , in chemotherapy-pretreated patients with castration-resistant prostate cancer. Clin Cancer Res. 2010;16:3028-34.

22. Fizazi K, De Bono JS, Fiechon A, et al. Randomised phase II study of siltuximab (CNTO 328) an anti-IL-6 monoclonal antibody, in combination with mitoxantrone/ prednisone versus mitoxantrone/prednisone alone in metastatic castration-resistant prostate cancer. Eur J Cancer. 2012;48:85-93.

23. Araki S, Omori Y, Lyn D, et al. Interleukin-8 is a molecular determinant of androgen independenceand progression in prostate cancer. Cancer Res. 2007;67:6854-62.

24. Luo Y, Azad AK, Karanika S, et al. Enzalutamide and CXCR7 inhibitor combination treatment suppresses cell growth and angiogenic signaling in castration-resistant prostate cancer models. Int JCancer. 2018;142:2163-74.

25. Hoefer J, Schäfer G, Klocker H, et al. PIAS1 is increased in human prostatecancer and enhances proliferation through inhibition of 21 . Am J Pathol. 2012;180:2097-107.

26. Puhr M, Santer F, Neuwirt H, et al. Down-regulation of suppressor of cytokine signaling-3 causes prostate cancer cell death through activation of the extrinsic and intrinsic apoptosis pathways. Cancer Res. 2009;69:7375-84.

27. Puhr M, Hoefer J, Eigentler A, et al. The glucocorticoid receptor is a key player for prostate cancer cell survival and a target for improved antiandrogen therapy. Clin Cancer Res. 2018;24:927-38.

28. Zhi ML, Kyprianou N. Role of androgens and the androgen receptor in epithelial-mesenchymal transition and invasion of prostate cancer cells. Faseb J. 2010;24:769-77.

29. Sun Y, Wang BE, Leong KG, et al. Androgen deprivation causes epithelial-mesenchymal transition in the prostate: Implications for androgen-deprivation therapy. Cancer Res. 2012;72:527-36.

30. Puhr M, Hoefer J, Schäfer G, et al. Epithelial-to-mesenchymal transition leads to docetaxel resistance in prostate cancer and is mediated by reduced expression of miR-200c and miR-205. Am J Pathol. 2012;181:2188-201.

31. Markowski MC, Silberstein JL, Eshleman JR, Eisenberger MA, Luo J, Antonarakis ES. Clinical utility of CLIA-grade ARV7 testing in patients with metastatic castration-resistant prostate cancer. JCO Precis Oncol. 2017; https://doi.org/ 10.1200/PO.17.00127.

32. Antonarakis ES, Chandhasin C, Osbourne E, Luo J, Sadar $\mathrm{MD}$, Perabo F. Targeting the $\mathrm{N}$-terminal domain of the androgen receptor: A new approach for the treatment of advanced prostate cancer. Oncologist. 2016;21:1427-35.

33. Graf JN, Alumkal JJ, Drake CG, et al. Early evidence of antiPD-1 activity in enzalutamide-resistant prostate cancer. Oncotarget. 2016;7:52810-7.

Publisher's Note Springer Nature remains neutral with regard to jurisdictional claims in published maps and institutional affiliations. 